\title{
Wer versteht wann was? Sprachverstehen im frühen Zweitspracherwerb des Deutschen am Beispiel der w-Fragen ${ }^{1}$
}

\begin{abstract}
Ob und welche kritischen Phasen für den Zweitspracherwerb existieren, wird im Hinblick auf Erwerbsverlauf und Erwerbsgeschwindigkeit kontrovers diskutiert. Für den Vergleich mit einsprachigen Lernern bietet der frühe Zweitspracherwerb ein ideales Untersuchungsfeld, da hier der Kontakt zur zweiten Sprache mit etwa drei Jahren, noch während des Erstspracherwerbs, einsetzt. Im vorliegenden Beitrag wird am Beispiel der w-Fragen untersucht, wie frühe Zweitsprachlerner die Interpretation von syntaktisch-semantisch komplexen Strukturen meistern. Die längsschnittlichen Daten wurden im Rahmen des Forschungsprojekts MILA mit dem Untertest w-Fragen aus LiSe-DaZ erhoben. Quantitative und qualitative Analysen belegen, dass sich die untersuchten frühen Zweitsprachlerner weder im Erwerbsverlauf noch in den Erwerbsmustern von den einsprachigen Lernern unterscheiden und dass sie ihnen in Bezug auf die Erwerbsgeschwindigkeit sogar überlegen sind. Für den w-Fragen-Erwerb ist folglich das Ende der kritischen Phase nicht vor dem dritten Geburtstag anzusetzen.
\end{abstract}

\section{Einleitung}

Antwortet ein Kind auf die Frage „Mit wem hast du heute gespielt?" mit "Ja“ oder „Verstecken“, kann der Fragende daraus schließen, dass das Kind die Interpretation dieses Frageformats noch nicht gemeistert hat. Solche Ergänzungs- oder Informationsfragen, im Folgenden w-Fragen genannt, spielen im Kindergarten- und Schulalltag eine wichtige Rolle: Sie dienen nicht nur der Strukturierung von Dialogen und dem Wissensaustausch, sondern auch der Wissensüberprüfung. Ihre zielsprachliche Interpretation lässt sich nur indirekt an den adäquaten Antworten ablesen, im obigen Beispiel etwa „mit Sara“ oder „mit ganz vielen Kindern“. Obgleich die menschliche Sprachkompetenz das Sprachverstehen ebenso wie die Sprachproduktion umfasst (Chomsky 1986), hat sich die (Erst-)Spracherwerbsforschung lange auf die Erforschung der Sprachproduktion anhand von Spontansprachkorpora konzentriert (Schulz/Grimm 2012). Dieser Fokus ist mit wenigen

Dieser Beitrag basiert auf Daten aus dem Projekt MILA, Center for Individual Development and Adaptive Education of Children at Risk (IDeA), das seit 2008 durch die LOEWE-Initiative der Hessischen Landesregierung gefördert wird. Mein Dank geht an die teilnehmenden Kinder und deren Eltern, an die beteiligten Institutionen sowie an die studentischen und wissenschaftlichen MitarbeiterInnen von MILA für die Unterstützung. 
Ausnahmen (z.B. Marinis/Chondrogianni 2011) auch für die Erforschung des Zweitspracherwerbs, sowohl für das Deutsche als auch für andere Sprachen, zu beobachten.

Im vorliegenden Beitrag zum frühen Zweitspracherwerb wird daher der Schwerpunkt auf das Sprachverstehen gelegt. Am Beispiel der w-Fragen wird untersucht, wie frühe Zweitsprachlerner, d.h. Kinder, die mit dem Erwerb des Deutschen im Alter zwischen 2 und 4 Jahren beginnen, im Vergleich mit einsprachigen Altersgenossen die zielsprachliche Interpretation von syntaktisch-semantisch komplexen Strukturen meistern. Im Rahmen des längsschnittlich angelegten Projekts MILA (The Role of Migration Background and Language Impairment in Children's Language Achievement) wurde das w-Frage-Verstehen mittels eines standardisierten Tests (Untertest WF aus dem Sprachtest LiSe-DaZ von Schulz/Tracy 2011) erhoben. Für den vorliegenden Beitrag wurden die längsschnittlichen Daten von 31 monolingualen Kindern und 17 frühen Zweitsprachlernern aus der MILA-Stichprobe über einen Zeitraum von zwei Jahren anhand von fünf Testzeitpunkten analysiert.

In Abschnitt 2 werden kindlicher Erst- und Zweitspracherwerb aus generativer Perspektive einander gegenüber gestellt; die Klassifikation nach Spracherwerbstyp wird von der Klassifikation nach Migrationshintergrund abgegrenzt. Abschnitt 3 fasst den Forschungsstand zum frühen Zweitspracherwerb des Deutschen zusammen. Während zur Sprachproduktion verschiedene Studien - meist basierend auf Spontansprachanalysen - vorliegen, stand das Sprachverstehen der frühen Zweitsprachlerner bis dato nur selten im Mittelpunkt der Forschung. In Abschnitt 4 wird der Gegenstand der vorliegenden Untersuchung skizziert: Nach der Beschreibung der Struktur einfacher w-Fragen werden die bisherigen Befunde zur Produktion und zum Verstehen von w-Fragen in Erst- und Zweitspracherwerb zusammengefasst. Abschnitt 5 bildet mit der Beschreibung der Methode, der Untersuchungsfragen, der Probanden und der Ergebnisse den empirischen Kern des vorliegenden Beitrags. In Abschnitt 6 werden die Ergebnisse vor dem Hintergrund der Untersuchungsfragen und der bisherigen Forschung diskutiert. Der Beitrag endet in Abschnitt 7 mit einem Fazit für Wissenschaft und Bildungspolitik.

\section{Varianten des Spracherwerbs}

\subsection{Spracherwerb, generell, generativ}

Der Erwerb einer oder mehrerer Sprachen stellt eine der grundlegendsten menschlichen Fähigkeiten dar. Neuere Erklärungsansätze gehen davon aus, dass der Sprachlerner dabei eine eigenaktive Rolle einnimmt, die es ihm erlaubt, aus dem sprachlichen Input die Strukturen der Umgebungsspra- 
che zu erschließen (siehe Tracy 2008; Schulz/Grimm 2012). Eine explizite Unterweisung durch die Bezugspersonen findet dabei nicht statt, d.h. der Spracherwerb findet ungesteuert statt. Dass der Spracherwerb in der Mehrzahl aller Fälle erfolgreich ist und unter unterschiedlichen Umweltbedingungen gelingt, liegt generativen Forschungsansätzen zufolge daran, dass der Sprachlerner eine sprachspezifische Prädisposition besitzt. Diese manifestiert sich durch das angeborene spezifisch menschliche Sprachwissen - die Universalgrammatik - sowie durch sprachspezifische Erwerbsstrategien. Sowohl die Zugänglichkeit zur Universalgrammatik und zur einzelsprachlichen Fixierung der Parameter als auch die Wirksamkeit der Spracherwerbsstrategien gilt als zeitlich begrenzt. Um muttersprachliche Kompetenz zu erwerben, ist daher aus generativer Sichtweise neben dem - qualitativ und quantitativ ausreichenden - sprachlichen Input ein Erwerbsbeginn innerhalb eines bestimmten Zeitfensters, der kritischen oder sensiblen Phase, notwendig (Tracy 2000; Meisel 2009). Neben dem Erstspracherwerb bietet insbesondere der Zweitspracherwerb ein ideales Untersuchungsfeld, um Relevanz und Dauer der kritischen Phase sowie die Wirksamkeit von Mechanismen zum Erwerb grammatischer Kompetenzen zu untersuchen (vgl. Unsworth 2005).

In der generativen Spracherwerbsforschung wird davon ausgegangen, dass das Sprachsystem ein von anderen kognitiven Fähigkeiten unabhängiges Modul darstellt; der Spracherwerb verläuft weitgehend unabhängig von der non-verbalen kognitiven Entwicklung. Zudem wird angenommen, dass das Sprachmodul selbst modular aufgebaut ist, so dass beispielsweise der Aufbau der einzelnen Teilsysteme der Grammatik wie Lexikon oder Syntax unterschiedlichen Erwerbsstrategien folgen kann.

Alle hier genannten Charakteristika gelten unabhängig davon, ob der Sprachlerner eine oder mehrere Sprachen erwirbt. Welche besonderen Eigenschaften den frühen Zweitspracherwerb auszeichnen, ist Gegenstand des folgenden Abschnitts.

\subsection{Früher Zweitspracherwerb, Migrationshintergrund und Migrantendeutsch}

Im Erstspracherwerb ist das chronologische Alter des Lerners zwangsläufig identisch mit der Kontaktdauer, d.h. mit der Länge des systematischen Kontakts mit der Zielsprache. Bei bilingualen Kindern dagegen fällt die Kontaktdauer nicht automatisch mit dem chronologischen Alter zusammen, sondern wird durch das Alter bei Erwerbsbeginn bestimmt. Beim simultan bilingualen Erwerb beginnt der Erwerb einer weiteren Sprache ab Geburt oder bis vor dem zweiten Geburtstag (de Houwer 1995; Tracy/GawlitzekMaiwald 2000). Im Zweitspracherwerb dagegen liegt der Erwerbsbeginn zwischen zwei Jahren und dem Erwachsenenalter. Die Differenzierung des 
Zweitspracherwerbs in verschiedene Subtypen beruht auf der Annahme, dass erhebliche Unterschiede im Erwerbsalter zu jeweils eigenen Gesetzmäßigkeiten für Erwerbsverlauf und -erfolg führen, die ggf. mit der Existenz von kritischen Phasen zu erklären sind. Sowohl über die Anzahl der Spracherwerbssubtypen als auch über die jeweiligen Altersgrenzen herrscht noch weitgehend Unklarheit. So wird häufig zwischen einem Erwerbsalter zwischen 2 bis 7 Jahren und später als 7 Jahren unterschieden (z.B. Krashen 1973; Hyltenstam 1992; Nicholas/Lightbown 2008). In jüngeren Studien wird innerhalb des Vorschulalters noch einmal nach einem Erwerbsbeginn vor oder nach dem vierten Geburtstag differenziert (Schwartz 2003; Tracy 2008; Meisel 2009; Rothweiler 2009; Schulz/Grimm 2012). Diesem Ansatz folgend wird als früber Zweitspracherwerb der Erwerb einer zweiten Sprache zwischen dem zweiten und vierten Geburtstag bezeichnet und als kindlicher Zweitspracherwerb ein Erwerb mit einem Erwerbsbeginn deutlich nach dem vierten Geburtstag. Sofern es sich um die Zielsprache Deutsch handelt und das Erwerbsalter erkenntlich ist, wird im Folgenden auch vereinfacht von Kindern mit DaZ (Deutsch als Zweitsprache) im Gegensatz zu Kindern mit DaM (Deutsch als Muttersprache) die Rede sein.

Frühe Zweitsprachlerner zeichnen sich dadurch aus, dass sie, anders als Erstsprachlerner, zum Zeitpunkt des ersten Kontakts mit der Zweitsprache bereits auf erworbenes Sprachwissen aus ihrer Erstsprache zurückgreifen können und zudem über vielfältige kognitive Fähigkeiten und Weltwissen verfügen. Ungeklärt ist, ob der frühe Zweitspracherwerb noch in die kritische Phase fällt und ob die Erwerbsmechanismen, die den Erstspracherwerb ermöglichen, noch aktiv sind. Offen ist zudem, ob und in welcher Weise beim frühen Zweitspracherwerb, wie für den Zweitspracherwerb Erwachsener belegt (vgl.White 2000), ein Transfer aus der Erstsprache erfolgt, der sich dann je nach Erstsprache begünstigend oder erschwerend auf den Erwerb der Struktur in der Zweitsprache auswirkt.

Bisherige Studien zum frühen Zweitspracherwerb beschränkten sich vor allem auf die morpho-syntaktische Entwicklung in Bezug auf die Sprachproduktion. Daher ist offen, inwiefern die Ergebnisse spezifisch für die untersuchte Modalität und den untersuchten Bereich sind, - zumal sich zunehmend Evidenz für die Existenz multipler kritischer Phasen für die verschiedenen sprachlichen Kernbereiche wie Phonologie, Morphologie, Syntax und Lexikon findet (z.B. Long 1990; Locke 1997; Bialystok 1997; Schwartz 2003).

Aus dieser Forschungslage ergeben sich zwei zentrale Aspekte, die für die Charakterisierung des frühen Zweitspracherwerbs zentral sind und deren Untersuchung einen Beitrag zur Frage der kritischen Phase(n) leisten kann: (a) die Qualität des Erwerbs und (b) die Erwerbsgeschwindigkeit. Der Aspekt der Qualität des Erwerbs umfasst die Fragen danach, ob die Erwerbsstufen und ihre Abfolge in Erst- und frühem Zweitspracherwerb gleich 
sind und ob die Fehlermuster in den jeweiligen Entwicklungsphasen den gleichen Regeln unterliegen. Dabei gilt es Analysekriterien für Gleichheit festzulegen und die interindividuelle Variation innerhalb der Gruppe der frühen Zweitsprachlerner in Bezug auf Kontaktdauer und Alter bei Erwerbsbeginn zu berücksichtigen. Aufgrund der grundsätzlichen Dissoziation zwischen Performanz und interner grammatischer Repräsentation sollte zudem von beobachteten Unterschieden bzw. Übereinstimmungen nicht zwangsläufig auf das grammatische System geschlossen werden (siehe Schulz 2012). Der zweite Aspekt der Erwerbsgeschwindigkeit beinhaltet zum einen die Frage, ob und wann - d.h. nach welcher Kontaktdauer und in welchem Alter - eine Struktur im Vergleich zum monolingualen Erwerb beherrscht wird, und zum anderen, wie schnell jeweils einzelne Erwerbsstufen erreicht werden. Auch hier stellt die interindividuelle Variation innerhalb der Zweitsprachlerner eine Herausforderung für die Vergleichbarkeit der Ergebnisse dar.

Generell stellt sich die Frage, welche Kriterien eine adäquate Vergleichsgruppe zu einer Gruppe von monolingualen Sprachlernern erfüllen sollte. In linguistischen Studien werden in erster Linie sprachliche Faktoren wie chronologisches Alter, Kontaktdauer und Alter bei Erwerbsbeginn kontrolliert, während nicht-sprachliche Faktoren wie Geschlecht, nonverbale Intelligenz und Bildungshintergrund weniger Berücksichtigung finden (z.B. Tracy/ Thoma 2009; Schönenberger/Rothweiler/Sterner 2012). Dagegen verwenden psychologische und sozialwissenschaftliche Studien, die nicht-sprachliche Faktoren typischerweise sorgfältig kontrollieren, häufig eine Klassifikation der Lerner nach Migrationshintergrund, d.h. danach, ob ein oder beide Elternteile außerhalb Deutschlands geboren wurden, statt nach Spracherwerbstyp. Die in diesen Studien häufig berichteten schlechteren sprachlichen Leistungen der Kinder mit Migrationshintergrund gegenüber Kindern ohne Migrationshintergrund (z.B. IGLU, Bos et al. 2007; PISA, Klieme et al. 2010) werden folglich auf den Migrationsstatus selbst zurückgeführt anstatt auf die häufig unterschiedliche Erwerbsbiographie der Kinder mit Migrationshintergrund. Viele der Kinder mit Migrationshintergrund haben den ersten systematischen Kontakt zum Deutschen mit Eintritt in den Kindergarten (vgl. Schulz/Tracy 2011) und damit ca. drei Jahre später als die typischerweise einsprachig aufwachsenden Kinder ohne Migrationshintergrund. ${ }^{2}$ Dass diese Kinder in altersparallelisierten Studien häufig besser abschneiden als gleichaltrige Kinder mit Migrationshintergrund kann daher aus linguistischer Sicht nicht überraschen. Erschwerend kommt hinzu, dass das Merkmal Migrationshintergrund nicht mit dem Spracherwerbstyp früher Zweitspracherwerb identisch ist. Dies zeigen Beispiele von einsprachig aufwachsenden Kindern mit einem im Ausland geborenen Elternteil ebenso

Die unselegierte Rekrutierungsstichprobe für das Projekt MILA ( $\mathrm{n}=600)$ im Raum Frankfurt beispielsweise umfasste zu $60 \%$ mehrsprachige Kinder; davon waren eine Hälfte frühe Zweitsprachlerner und die andere Hälfte simultan bilingual aufwachsende Kinder. 
wie Kinder, deren Eltern beide in Deutschland geboren sind und die trotzdem Deutsch als frühe Zweitsprache erwerben (vgl. Rauch/Grimm/Schulz eingereicht). Folglich sind Migrationshintergrund und damit auch Migrantendeutsch für die Spracherwerbsforschung keine sinnvollen Kategorien.

Wenn es darum geht, verlässliche Aussagen über die Sprachentwicklung und die sprachlichen Fähigkeiten von kindlichen Lernern zu gewinnen, ist aus linguistischer Perspektive eine Klassifikation nach Spracherwerbstyp, die Kontaktdauer und Alter bei Erwerbsbeginn umfasst, anstatt nach Migrationshintergrund zwingend erforderlich. Von der sozialwissenschaftlichen Forschung ließe sich lernen, nicht-sprachliche Faktoren wie nonverbale Intelligenz und Bildungshintergrund, in denen sich in den oben genannten Studien Kinder mit und ohne Migrationshintergrund häufig gravierend unterscheiden, stärker zu berücksichtigen.

\section{Was wir über den frühen Zweitspracherwerb (nicht) wissen}

\subsection{Sprachproduktion}

Der frühe Zweitspracherwerb des Deutschen wird erst seit Anfang der 2000er Jahre intensiv aus sprachwissenschaftlicher Perspektive erforscht (vgl. Ahrenholz (Hg.) 2006). Der Tradition der Spracherwerbsforschung folgend liegt der Fokus auf der Sprachproduktion und insbesondere auf der MorphoSyntax (vgl. Schulz/Grimm 2012). In längsschnittlich angelegten Spontansprachstudien wurden anhand kleiner Stichproben vor allem Kinder mit Türkisch, Arabisch und Russisch als Erstsprache untersucht. Neben Kasus und Genus (Lemke 2009; Hopp 2011; Schönenberger/Rothweiler/Sterner 2012; Tracy/Lemke 2012) stand vor allem der Erwerb von Verbstellung, Verbflexion und Subjekt-Verb-Kongruenz (Haberzettl 2005; Rothweiler 2007; Thoma/Tracy 2006; Chilla 2008; Tracy/Thoma 2009) im Mittelpunkt der Forschung.

Die Ergebnisse dieser Studien zeigen, dass bei einem Erwerbsbeginn zwischen zwei und vier Jahren in Bezug auf den Erwerbsverlauf und die Erwerbsmuster große Parallelen zum Erstspracherwerb bestehen. In Bezug auf die Erwerbsgeschwindigkeit gibt es zudem Belege dafür, dass - vermutlich bedingt durch das bereits vorhandene grammatische Wissen aus der Erstsprache - bestimmte Entwicklungsschritte sogar schneller durchlaufen werden als im monolingualen Erwerb des Deutschen (Rothweiler 2007; Thoma/ Tracy 2006; Tracy/Thoma 2009). Zu Beginn werden Mehrwortäußerungen mit unflektierten Verben in der rechten Satzklammer produziert. Nach 6 bis 12 Kontaktmonaten werden Verbzweit-Stellung und Subjekt-Verb-Kongruenz realisiert. Flektierte Verben werden zielsprachlich in VerbzweitPosition produziert und kongruieren mit dem Subjekt (modulo kleinere vor allem verbspezifische Abweichungen); auch w-Fragen mit overtem w-Pro- 
nomen werden in dieser Phase analog zum Erstspracherwerb produziert. Der Erwerb von Nebensätzen erfolgt nach 12 bis 18 Kontaktmonaten. Wie im Erstspracherwerb sind im frühen Zweitspracherwerb nicht-flektierte Verben in der Verbzweit-Position und mehrteilige Verbgefüge wie habe gesehen in der linken Satzklammer sehr selten zu beobachten. ${ }^{3}$ Einschränkend ist festzuhalten, dass diese Ergebnisse auf der Auswertung von Spontansprachkorpora basieren und die beispielsweise von Demuth (1996) geforderte Absicherung durch ergänzende experimentelle Untersuchungen nicht erfüllen. Pilotergebnisse zum Untertest Sprachproduktion aus dem standardisierten Test LiSe-DaZ sowie die Normierungsergebnisse bestätigen den raschen Erwerb der Satzklammer anhand von Querschnittsdaten (Schulz/ Tracy/Wenzel 2008; Schulz/Tracy 2011).

\subsection{Sprachverstehen}

Die zielsprachliche Produktion einer Struktur geht nicht notwendigerweise mit deren korrekter Interpretation einher. Diese Dissoziation zwischen Produktion und Verstehen wurde im Erstspracherwerb anhand von Studien zum Wortschatz und zu verschiedenen syntaktischen Strukturen untersucht (vgl. Schulz 2007). Im Gegensatz zur Sprachproduktion ist das Sprachverstehen im frühen Zweitspracherwerb generell noch wenig erforscht (siehe jedoch Marinis/Chondrogianni 2011 für türkisch-englische Zweitsprachlerner). Eine der ersten Untersuchungen zum Deutschen als Zweitsprache stammt von Penner (1998), der Defizite bei Ausländerkindern in der deutschen Schweiz, z.B. im Bereich der Nominalphrase, feststellte. Im pädagogischen Kontext konstatierte Knapp (1999) bei Kindern mit Deutsch als Zweitsprache das Problem verdeckter Sprachschwierigkeiten, die er u.a. auf Verstehensprobleme zurückführt, welche in der Alltagskommunikation häufig nicht auffallen. Erste detailliertere Studien zur Interpretation von semantischen Phänomen wie anaphorischen Ausdrücken (Ose/Schulz 2010), exhaustiven w-Fragen und quantifizierten Sätzen (vgl. Schulz/Grimm 2012) weisen insgesamt darauf hin, dass die frühen Zweitsprachlerner auf dem Weg zur zielsprachlichen Interpretation prinzipiell den gleichen Erwerbsverlauf und ähnliche Erwerbsmuster wie im Erstspracherwerb aufweisen. In Bezug auf die Erwerbsgeschwindigkeit wurde - bei einem Erwerbsbeginn mit 3 Jahren - generell ein Abstand von etwa einem Jahr gegenüber den monolingualen Kindern festgestellt, was wie in der Sprachproduktion gleichbedeutend mit einem schnelleren Durchlaufen der Erwerbsphasen wäre. Einschränkend ist hinzuzufügen, dass die Studien jeweils als Querschnittsdesign mit wenigen Altersgruppen angelegt waren. Zur Absiche-

\footnotetext{
Bei einem späteren Erwerbsbeginn zwischen 6;0 und 8;0 sind diese Strukturen (z.B. Ich habe gesehen die Batterie), die an den Zweitspracherwerb Erwachsener erinnern, jedoch dokumentiert (Haberzettl 2005).
} 
rung der Ergebnisse wären aufgrund der größeren Variation innerhalb der Gruppe der frühen Zweitsprachlerner zusätzlich Längsschnittstudien hilfreich, die es erlauben, die Veränderung der Lernergrammatiken innerhalb eines Individuums nachzuzeichnen.

\section{W-Fragen}

\subsection{Struktur von w-Fragen}

W-Fragen, die auch als Ergänzungs- oder Informationsfragen bezeichnet werden, zählen zu den im Sprachangebot besonders häufigen Satztypen. Während Aussagen wie „Max hilft dem Hund“ eine Proposition ausdrücken, drücken Fragen mehrere alternative Propositionen aus. Entscheidungsfragen wie „Hilft Max dem Hund?“ sind mit einer Situation, in der Max dem Hund hilft oder nicht hilft, kompatibel und fordern als Antwort folglich je nach Situation ja oder nein. Bei w-Fragen wie „Wem hilft Max?" besteht die adäquate Antwort aus der Liste der Individuen, für die gilt, dass Max ihnen hilft, beispielsweise \{Fritz, Moritz, Otto $\}$. Ob diese so genannte exhaustive Lesart jeweils der Default ist oder aufgrund pragmatischer Inferenzen zustande kommt, ist strittig (vgl. die Diskussion in Schulz/Roeper 2011). Besteht die Liste lediglich aus einem Element wie $\{$ Moritz $\}$, ist die exhaustive Antwort gleichbedeutend mit dieser Einer-Liste. Im Folgenden wird nur auf w-Fragen eingegangen, die ein w-Pronomen enthalten und bei denen die exhaustive Antwort eine Einer-Liste ist. Für die Erörterung multipler w-Fragen wie „Wer hilft wem?““ sei auf Schulz/Roeper (2011) verwiesen.

W-Fragen werden im Deutschen durch w-Bewegung gebildet, d.h. genau ein w-Fragepronomen wird satzinitial in eine Nicht-Argument-Position bewegt, wie in (1a) illustriert. Neben dieser overten w-Bewegung muss das Verb in eine Position bewegt werden, die das Merkmal [+w] tragen kann, um das so genannte wh-Criterion (Rizzi 1996) zu erfüllen, das zwischen wOperator und Träger des w-Merkmals eine Spezifikator-Kopf-Relation erfordert. Die Frage, welche Spezifikatorposition das w-Pronomen aufnimmt, wird hier nicht erörtert, da sie für die weitere Diskussion keine Rolle spielt. ${ }^{4}$

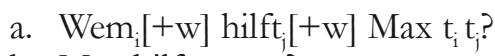
b. Max hilft WEM?

Verbleibt das w-Pronomen wie in (1b) in situ, ergibt sich im Deutschen nur die Möglichkeit der Lesart als Echofrage. In w-in-situ-Sprachen (z.B. Türkisch, Chinesisch) werden w-Fragen ohne satzinitiale Bewegung des w-Pronomens gebildet (Dayal 2002).

$4 \quad$ Guasti (2002) zufolge bewegt sich das w-Pronomen in die SpecCP; siehe jedoch Grewendorf (2002, S. 235) für Argumente zugunsten der Nicht-Operatorposition SpecFinP. 
W-Fragen lassen sich nach der Art der bewegten Konstituente als Argumentfragen (2a-c) oder Adjunktfragen (2d) klassifizieren.

(2) a. Wer ${ }_{i}$ hilft $t_{i}$ dem Hund?

b. Wen füttert Max $t_{i}$ ?

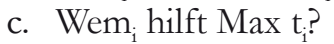

d. $\operatorname{Wann}_{\mathrm{i}} / \mathrm{wo}_{\mathrm{i}} /$ warum $_{\mathrm{i}}$ füttert Max den Hund $\mathrm{t}_{\mathrm{i}}$ ?

Während Argumentfragen jeweils eine Konstituente erfragen, die durch die thematische Rolle des Verbs obligatorisch ist (Subjekt (2a), Akkusativobjekt (2b) bzw. Dativobjekt (2c)), ist die erfragte Konstituente in Adjunktfragen wie (2d) nicht durch das Thetaraster des Verbs vorgegeben.

\subsection{Erwerb von w-Fragen im Erst- und Zweitspracherwerb}

Im monolingualen Spracherwerb werden erste Fragestrukturen mit w-FrageBedeutung ohne overtes w-Pronomen (3) bereits mit ca. 20 Monaten produziert, gefolgt von w-Fragen mit overtem w-Fragepronomen (4) (Penner 1994; Tracy 1994). Bis etwa zum dritten Geburtstag werden die verschiedenen Fragepronomen in den aktiven Wortschatz aufgenommen, in der Reihenfolge wo $/$ was $<$ wer $<$ wie $<$ warum $<$ wann.

(3) a. Der Flöte is?

b. Isn das?

(4) a. Wo ist Max?

b. Warum der auch ein Keks isst?

Auf dem Weg zur zielsprachlichen Struktur sind neben Verbzweit-Formaten (4a) auch w-Fragen mit Verbend-Stellung (4b) zu beobachten (Tracy 1994). Jedoch gibt es keine Evidenz für w-Fragen, in denen das w-Pronomen in situ, d.h. in der Basisposition, verbleibt (Guasti 2002) wie in „der auch ein Keks isst warum“.

Das Verstehen von w-Fragen stellt eine komplexe Lernaufgabe dar, da der Lerner nicht nur erkennen muss, dass nach einem im Satz nicht realisierten Satzglied gefragt wird, sondern auch, um welches Satzglied es sich handelt. Jüngere Kinder antworten auf w-Fragen zu Beginn wie auf Entscheidungsfragen mit $j a$ oder nein, was darauf hindeutet, dass sie das wPronomen nicht interpretieren. In einem nächsten Erwerbsschritt wird die w-Frage mit einer Konstituente beantwortet, die jedoch nicht immer dem erfragten Satzglied entspricht (Siegmüller/Herzog/Herrmann 2005). Die zielsprachliche Interpretation von w-Fragen gelingt im Alter von ca. 4 Jahren (Penner 1999; Schulz/Tracy/Wenzel 2008). In experimentellen Studien zu verschiedenen Sprachen zeigte sich zudem, dass die zielsprachliche Interpretation von Argumentfragen eher erworben wird als die von Adjunktfragen und dass innerhalb der Argumentfragen Subjektfragen eher als Objektfragen zielsprachlich interpretiert werden (Friedmann/Novogrodsky 2011). 
Der Erwerb von w-Fragen bei frühen Zweitsprachlernern stand bis dato nur selten explizit im Fokus von Erwerbsstudien. Im Rahmen der Untersuchung der Produktion von Verbzweit-Strukturen finden sich Belege dafür, dass erste w-Strukturen bereits die zielsprachliche Verbbewegung aufweisen, wie die w-Fragen in (5), die beide von einem Kind nach ca. 6 Kontaktmonaten geäußert wurden (Tracy/Lemke 2012). Lediglich die nicht-zielsprachliche Verwendung des Fragepronomens wenn statt wann und die inkorrekte Kasusmarkierung des definiten Artikels in (5b) sind auffällig.
a. Warum hast du des?
b. Wenn gehen wir in der Gruppe?
RNV (3;5 Jahre, ca. 8 KM)

Eine Spontansprachanalyse von acht frühen Zweitsprachlernern mit Türkisch als Erstsprache und einem Alter bei Erwerbsbeginn zwischen 2;7 und 4;3 von Chilla/Bonnesen (2011) bestätigt, dass w-Fragen nach einem Jahr Kontaktdauer zielsprachlich gebildet werden und dass Abweichungen vor allem durch fehlendes Verb (6a) oder seltener durch fehlendes Subjekt (6b) entstehen. Lediglich bei einem Lerner (Alter bei Erwerbsbeginn: 4;3 Jahre) sind vereinzelt w-Fragen mit w-in-situ belegt (7). Ob dieser Unterschied tatsächlich auf das Erwerbsalter $>$ 4;0 zurückzuführen ist, wie Chilla/Bonnesen (2011, S. 430) behaupten, scheint angesichts der schmalen Datenbasis jedoch fraglich.
a. Was das?
b. is das?

Eser (3;11 Jahre, $11 \mathrm{KM})$

Eser (3;11 Jahre, $11 \mathrm{KM})$

Fikret (4;11 Jahre, $8 \mathrm{KM})$

Fikret (5;06 Jahre, $15 \mathrm{KM})$

Untersuchungen zur Interpretation einfacher w-Fragen bei frühen Zweitsprachlernern ergaben bis dato widersprüchliche Ergebnisse. Penner (2003) zufolge weisen Kindergartenkinder mit Deutsch als Zweitsprache gegenüber monolingualen Kindern gravierende Probleme mit w-Fragen auf (47\% gegenüber 85\% korrekte Antworten). Andererseits zeigten Querschnittsdaten einer Pilotversion des Sprachtests LiSe-DaZ (Schulz/Tracy 2011, siehe Abschnitt 5.4) mit 88 drei- bis sechsjährigen Kindern mit Deutsch als Zweitsprache, dass das zielsprachliche Verständnis von w-Fragen mit etwa sechs Jahren bzw. nach 26 Kontaktmonaten erworben wird (Schulz/Tracy/Wenzel 2008; Wenzel/Schulz/Tracy 2009). Offen ist folglich zum einen, wann und wie schnell $\mathrm{w}$-Fragen zielsprachlich interpretiert werden und zum anderen, wie der Erwerbsverlauf im Vergleich zu monolingualen Lernern aussieht. Offen ist darüber hinaus, welchen Einfluss nicht-sprachliche Faktoren wie Bildungshintergrund und nonverbale Intelligenz auf das w-Frage-Verstehen besitzen. Diesen Fragen wurde im Rahmen des Forschungsprojekts MILA in einer Längsschnittstudie nachgegangen. 


\section{Empirische Studie: Interpretation einfacher w-Fragen}

\subsection{Hintergrund der Studie: Das Projekt MILA}

In dem Projekt MILA wird die Sprachentwicklung bei Kindern mit DaM und DaZ, jeweils mit und ohne spezifische Sprachentwicklungsstörungen (SSES), untersucht. Insgesamt werden je $60 \mathrm{DaM}$ - und 60 DaZ-Kinder in einem kombinierten Längs- und Querschnittsdesign mittels unterschiedlicher Methoden (standardisierte Testverfahren, Spontansprache, psycholinguistische Experimente) über einen Zeitraum von fünf Jahren begleitet (siehe Grimm/Schulz 2012). Vor dem Hintergrund der in Abschnitt 2 skizzierten Forschungslage verfolgt MILA das Ziel, die Sprachkompetenz früher Zweitsprachlerner des Deutschen umfassend zu charakterisieren und dabei insbesondere bis dato weniger erforschte Bereiche wie das Sprachverstehen und den Erwerb semantischer und phonologischer Fähigkeiten bei Kindern mit DaZ zu berücksichtigen. Aufgrund des Gruppendesigns lassen sich - anders als in bisherigen Fallstudien - sowohl der Einfluss externer Faktoren wie Bildungshintergrund auf die Sprachentwicklung untersuchen als auch Beziehungen zwischen den sprachlichen und nichtsprachlichen Fähigkeiten wie der nonverbalen Intelligenz bestimmen (vgl. Grimm et al. eingereicht). Das Längsschnittdesign ermöglicht es zudem, wie in den erwähnten Fallstudien individuelle Entwicklungsverläufe zu beschreiben. Mit diesem multimethodischen Ansatz schließt MILA an aktuelle Studien in Holland, Kanada und Israel an, in denen jeweils mehrsprachige Kinder mit sprachunauffälligem und sprachauffälligem Erwerbsverlauf anhand verschiedener Verfahren untersucht werden. Um eine möglichst große Vergleichbarkeit zwischen der DaZ- und der DaM-Gruppe zu erreichen, mussten die sprachunauffälligen Kinder bestimmte Einschluss-Kriterien erfüllen, die in Tabelle 1 zusammengefasst sind.

\begin{tabular}{ll}
\hline DaM & DaZ \\
\hline \multirow{3}{*}{ Monolingualer Erwerb des Deutschen } & $\begin{array}{l}\text { Nicht-deutsche Muttersprache } \\
\text { Beginn des Deutscherwerbs nach dem } \\
\text { 2. Geburtstag }\end{array}$ \\
\hline Altersentsprechender nonverbaler IQ & Altersentsprechender nonverbaler IQ \\
Normales Hörvermögen & Normales Hörvermögen \\
Keine Sprachtherapie & Keine Sprachtherapie \\
Altersgerechte T-Werte in LiSe-DaZ & Altersgerechte T-Werte in LiSe-DaZ \\
Alter zwischen 3;6 und 3;11 Jahre & Alter zwischen 3;6 und 3;11 Jahre \\
\hline
\end{tabular}

Tab. 1: Einschlusskriterien für die sprachunauffälligen Gruppen der MILA-Gesamtstichprobe zum ersten Testzeitpunkt

T-Wert $<40$ in höchstens einem Untertest. 
In Bezug auf die Erstsprache der DaZ-Kinder wurden keine weiteren Einschlusskriterien festgelegt. Innerhalb der DaZ-Gruppe sind insgesamt 18 verschiedene Erstsprachen vertreten, mit Türkisch $(\mathrm{N}=17)$, Serbisch und Kroatisch $(\mathrm{N}=4)$ und Arabisch $(\mathrm{N}=4)$ als den häufigsten Sprachen.

\subsection{Untersuchungsfragen}

Ob der frühe Zweitspracherwerb noch in die Phase fällt, in der der Spracherwerb nach den gleichen Gesetzmäßigkeiten und ähnlich erfolgreich wie der Erstspracherwerb verläuft, ist nach wie vor ungeklärt. Ziel der vorliegenden Studie ist es, einen Beitrag zur Beantwortung dieser Fragen zu leisten. Folgende spezifische Fragen zu Erwerbsgeschwindigkeit und Qualität stehen im Mittelpunkt unserer Studie:

a. Geschwindigkeit des Erwerbs

- Wie schnell werden die einzelnen Erwerbsstufen in der Interpretation von w-Fragen erreicht?

- Wann wird die w-Frage-Struktur zielsprachlich interpretiert?

b. Qualität des Erwerbs

- Sind die Erwerbsstufen und ihre Abfolge gleich?

- Unterliegen die Fehlermuster in der jeweiligen Entwicklungsphase den gleichen Regeln?

Aufgrund des relativ niedrigen Alters bei Erwerbsbeginn wird erwartet, dass die frühen Zweitsprachlerner die Interpretation von w-Fragen ähnlich schnell und in ähnlicher Weise wie Erstsprachlerner meistern, d.h. nach ca. drei Jahren Kontakt mit der Zielsprache, wobei die Interpretation von Argumentfragen leichter fallen sollte als die von Adjunktfragen. W-Fragen gehören zu den kerngrammatischen regelgeleiteten Bereichen der Sprache, die weniger vom konkret verfügbaren Sprachangebot der Umgebung, sondern eher von den angeborenen Strukturen für Frageformate abhängen. Daher sind Einflüsse nicht-sprachlicher Faktoren wie Bildungshintergrund und nonverbale Intelligenz nicht zu erwarten.

\subsection{Probanden}

Die Datengrundlage bilden MILA-Teilstichproben von 31 Kindern mit DaM (13 Mädchen, 18 Jungen) und 17 Kindern mit DaZ (9 Mädchen, 8 Jungen), für die für alle fünf Testzeitpunkte vollständige Datensätze vorliegen. Das Durchschnittsalter dieser DaM-Gruppe betrug zum ersten Erhebungszeitpunkt 3;8 Jahre (44.6 Monate; SD = 2.2). Die DaZ-Gruppe war zum ersten Erhebungszeitpunkt ebenfalls 3;8 Jahre alt (44.3 Monate; SD = 2.0) und hatte im Durchschnitt 10.2 Monate Kontakt zum Deutschen (SD = 4.5; 5-19 Monate). Das Alter bei Erwerbsbeginn lag durchschnittlich bei 2;8 
Jahren (24-41 Monate). Innerhalb der DaZ-Gruppe sind neun verschiedene Erstsprachen vertreten, wobei Türkisch $(\mathrm{N}=6)$, Serbisch und Kroatisch $(\mathrm{N}=3)$, Russisch $(\mathrm{N}=2)$ und Persisch $(\mathrm{N}=2)$ die am häufigsten genannten Sprachen darstellen.

Die nonverbalen kognitiven Fähigkeiten wurden im Alter von 4;2 Jahren $(\mathrm{DaM}=50$ Monate, $\mathrm{SD}=1.9 ; \mathrm{DaZ}=50.4$ Monate, $\mathrm{SD}=1.7)$ mit Hilfe der nonverbalen Untertests „Wiedererkennen von Gesichtern“, „Handbewegungen“ und „Dreiecke“ aus dem K-ABC (Melchers/Preuss 2003) untersucht. Sowohl in der DaM- als auch in der DaZ-Gruppe liegen die Testwerte in den nonverbalen Subtests des K-ABC über dem kritischen Wert von 70 Punkten (DaM: 90.7 Punkte, SD = 13.3; DaZ: 84.6 Punkte, SD = 14.4). Damit weisen alle Kinder eine altersentsprechende kognitive Intelligenz auf. Die Unterschiede zwischen der DaM- und der DaZ-Gruppe sind nicht signifikant (Mann-Whitney-U-Test, $z=-1.362 ; p=.173$ ).

Als Maß für den Bildungshintergrund wurde mittels Fragebogen die Dauer der Schulbildung von Vater und Mutter erhoben. Im Durchschnitt besuchten die Mütter in der DaM-Gruppe die Schule 11.3 Jahre (SD = 1.6) und die Väter 10.9 Jahre (SD = 2.7). In der DaZ-Gruppe betrug die Schulbesuchsdauer der Mütter durchschnittlich $8.6(\mathrm{SD}=3.9)$ und die der Väter 9.5 Jahre $(\mathrm{SD}=3.5)$. Erwartungsgemäß zeigte sich, dass die Mütter der DaZ-Kinder eine signifikant kürzere Schulbesuchsdauer aufweisen als die Mütter der DaM-Kinder; für die Väter war der Unterschied nicht signifikant (Mütter: $p=.014$; Väter: $p=.124$, T-Test).

\subsection{Methode}

Die Interpretation von w-Fragen wurde anhand des Untertests WF aus dem standardisierten Sprachtest LiSe-DaZ (Schulz/Tracy 2011) erfasst. Um die längsschnittliche Entwicklung abzubilden, wurden die Analysen jeweils auf der Grundlage der Rohwerte statt der T-Werte durchgeführt.

Der Untertest WF basiert auf der Methode der Questions-after-Story, die es erlaubt, die grammatische Kompetenz gezielt und ohne Einfluss von Kontexteffekten und Weltwissen des Kindes zu untersuchen (de Villiers/ Roeper 1996). Im Test WF wird dem Kind ein Bild gezeigt und die dort abgebildete Situation kurz beschrieben. Die Protagonisten sowie die Mehrzahl der Bilder entstammen dem Untertest zur Sprachproduktion, der anhand eines Bilderbuchs eine Abenteuergeschichte erzählt (siehe Schulz/ Tracy 2011, S. 33 ff.) und der jeweils vorher durchgeführt wurde. Nach der Bildbeschreibung stellt der Testleiter eine w-Frage, die sich auf ein Element des Bildes bzw. der Situation bezieht und die nur korrekt beantwortet werden kann, wenn die Struktur der w-Frage korrekt interpretiert wird, wie in Beispiel (9) und Abbildung (1) illustriert. Das Kind kann verbal mit der erfragten Konstituente antworten oder auf das entsprechende Objekt zeigen. 
(9) Testleiter: Ibo hilft dem Hund aus der Tonne. Er ist eingesperrt und kann nicht allein raus.

Testfrage: Wem hilft Ibo aus der Tonne?

Semantisch korrekte Antwort: Dem Hund/den Hund/Hund/das Hund.

Abweichende Antworten: Ja/die zwei/die Mülleimer/Tonne.

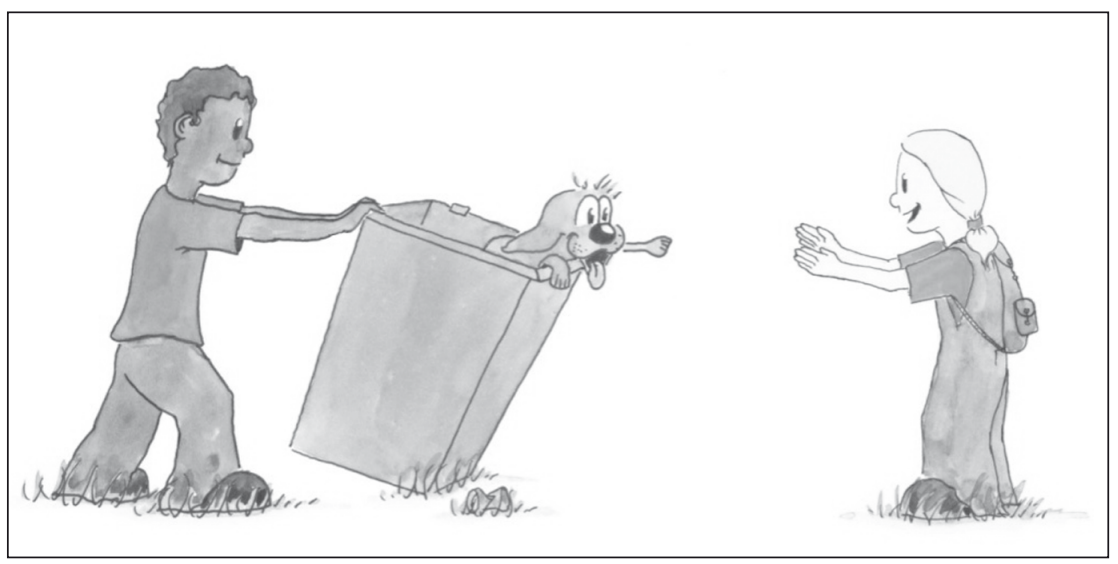

Abb. 1: Beispielitem für den Untertest WF

Insgesamt umfasst der Untertest WT neben zwei Übungsitems, die das Kind mit der Methode vertraut machen und die nicht in die Analyse eingehen, 10 Testitems: 6 Argumentfragen (2 Subjekt: wer, 4 Objekt: wen, wem) und 4 Adjunktfragen (womit, wann). Die Auswahl der fünf w-Fragepronomen erfolgte aufgrund der prototypischen Zugehörigkeit zu den einzelnen Fragetypen und der attestierten frühen Verwendung im aktiven kindlichen Wortschatz sowie auf den Ergebnissen mehrerer Pilotversionen.

Neben der quantitativen Auswertung wurden in einer zusätzlichen qualitativen Auswertung nicht-zielsprachliche Antworten nach der Art der Abweichung wie folgt klassifiziert: Keine Antwort, Ja/Nein-Antwort, w-Antwort mit zusätzlicher Konstituente (z.B. in (9) die zwei) oder mit abweichender Konstituente (z.B. in (9) Tonne). Die Reihenfolge der genannten Fehlertypen spiegelt dabei den Erwerbsfortschritt wider; so ist die Antwort mit einer abweichenden Konstituente fortschrittlicher als die Antwort mit Ja/Nein.

\subsection{Durchführung}

Alle Kinder nahmen im Abstand von ca. jeweils sechs Monaten an insgesamt fünf Erhebungen teil. Neben LiSe-DaZ wurden zu den verschiedenen Testzeitpunkten jeweils weitere Erhebungen durchgeführt. Alle Kinder wurden individuell von geschulten Projektmitarbeitern in der Kita bzw. auf 
Wunsch der Eltern zu Hause untersucht. Die Erhebungen wurden videographiert, wobei Erhebung und Auswertung jeweils durch unterschiedliche Mitarbeiterinnen erfolgte.

\subsection{Ergebnisse}

Erwerbsgeschwindigkeit. Um die Geschwindigkeit von DaM- und DaZ-Kindern im Erwerb der Interpretation von w-Fragen zu untersuchen, wurden für jeden Testzeitpunkt jeweils die Mittelwerte korrekter Antworten für die beiden Lernergruppen berechnet. Tabelle 2 fasst die statistischen Kennwerte und die Ergebnisse der MANOVA ${ }^{6}$ pro Testzeitpunkt zusammen.

\begin{tabular}{cccccc}
\hline Alter & \multicolumn{2}{c}{ DaM } & \multicolumn{2}{c}{ DaZ } & \multirow{2}{*}{ MANOVA } \\
\cline { 1 - 5 } & $M$ & \multicolumn{1}{c}{$S D$} & $M$ & \multicolumn{1}{c}{$S D$} & $\mathrm{p}$ \\
\cline { 2 - 5 } $3 ; 8$ & 74.19 & 18.93 & 32.94 & 24.69 & $.000^{* * *}$ \\
$4 ; 2$ & 84.51 & 14.10 & 59.42 & 24.87 & $.000^{* * *}$ \\
$4 ; 8$ & 89.36 & 8.14 & 76.47 & 20.90 & $.004^{* *}$ \\
$5 ; 2$ & 91.61 & 9.34 & 75.29 & 21.25 & $.001^{* *}$ \\
$5 ; 8$ & 91.29 & 9.22 & 86.47 & 11.15 & .115 \\
\hline
\end{tabular}

*** $p<.001, * * p<.01$

Tab. 2: Mittelwerte (M) und Standardabweichung (SD) der korrekten Antworten für die DaMund DaZ-Gruppe und MANOVA

Die DaM-Gruppe schneidet zu jedem Testzeitpunkt deskriptiv besser ab als die DaZ-Gruppe; diese Unterschiede sind für die ersten vier Testzeitpunkte signifikant. Legt man ein Mastery-Kriterium von rund $90 \%$ korrekten Antworten an, beherrschen die einsprachigen Lerner die Interpretation von w-Fragen im Alter von 4;8 und die frühen Zweitsprachlerner im Alter von ca. 5;8. Abbildung 2 illustriert die Entwicklungsverläufe beider Lernergruppen.

Während die DaM-Lerner w-Fragen bereits im Alter von 3;8 Jahren in knapp Dreiviertel aller Fälle beherrschen, gelingt dies der DaZ-Gruppe bei gleichem chronologischen Alter in lediglich einem Drittel der Fälle. Jedoch nehmen die Verstehensleistungen der DaZ-Lerner innerhalb eines Jahres sehr stark zu. Mit 4;8 erreichen sie die gleichen Leistungen wie die DaMGruppe mit 3;8 und haben im Alter von 5;8 ihre einsprachigen Altersgenossen fast eingeholt, - trotz der naturgemäß insgesamt wesentlich geringeren Kontaktdauer zum Deutschen $(\mathrm{DaM}=67$ Monate; DaZ $=34$ Monate $)$.

${ }_{6} \quad$ Multivariate Varianzanalyse für mehr als eine abhängige Variable. 
Daraus lässt sich schließen, dass die frühen Zweitsprachlerner in der Interpretation der w-Fragen insgesamt eine höhere Erwerbsgeschwindigkeit aufweisen als die einsprachigen Lerner.

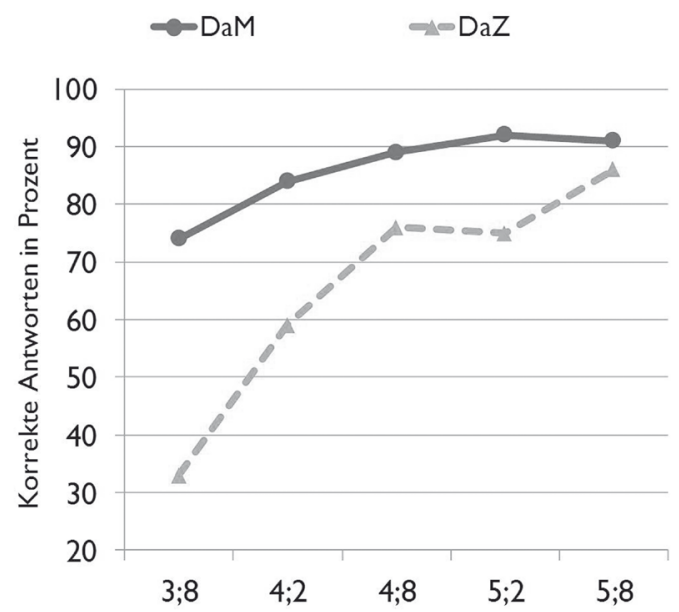

Abb. 2: Untertest w-Fragen. Prozentsatz zielsprachlicher Antworten für Kinder mit DaZ und DaM nach Alter

In einem nächsten Schritt wurden Subjekt-, Objekt- und Adjunkt-w-Fragen getrennt analysiert, um zu klären, ob auch innerhalb der drei w-Fragetypen eine höhere Erwerbsgeschwindigkeit zu beobachten ist. Tabelle 3 gibt einen Überblick über die statistischen Kennwerte und die Ergebnisse der MANOVA pro Testzeitpunkt.

Generell schneidet die DaM-Gruppe zu jedem Testzeitpunkt in allen drei Fragetypen deskriptiv besser ab als die DaZ-Gruppe (bis auf die Subjekt-w-Fragen mit 5;8 Jahren, wo beide Gruppen 100\% erreichen). In den Subjekt-w-Fragen ist die Verstehensleistung der DaZ-Gruppe zu den ersten zwei Testzeitpunkten signifikant schlechter als die der DaM-Gruppe. In den Objekt- und den Adjunkt-w-Fragen schneidet die DaZ-Gruppe jeweils zu den ersten vier Testzeitpunkten signifikant schlechter ab als die DaMGruppe. Legt man das Mastery-Kriterium von rund 90\% korrekten Antworten an, beherrschen die DaM-Kinder die Interpretation von Subjekt-w-Fragen bereits zum ersten Testzeitpunkt mit 3;8 und die DaZ-Kinder im Alter von 4;8, nach 22 Monaten Kontakt zum Deutschen. Objekt-w-Fragen werden von den DaM-Kindern mit 4;8 Jahren beherrscht und von den DaZKindern mit 5;8 Jahren, nach 34 Kontaktmonaten. Die Interpretation von Adjunkt-w-Fragen gelingt DaM- und DaZ-Kindern bis zum Alter von 5;8 noch nicht sicher. Wie für die w-Fragen insgesamt zeigt sich auch für die Subtypen, dass die Verstehensleistungen der DaZ-Lerner innerhalb des Untersuchungszeitraums sehr stark zunehmen. Jeweils innerhalb eines Jahres 
zeigen sie in den Subjekt-und Objekt-w-Fragen Leistungen, die mit denen ihrer einsprachigen Altersgenossen vergleichbar sind, - trotz der insgesamt wesentlich geringeren Kontaktdauer zum Deutschen.

\begin{tabular}{|c|c|c|c|c|c|c|}
\hline \multirow[t]{2}{*}{ Alter } & \multirow[t]{2}{*}{ Fragetyp } & \multicolumn{2}{|c|}{ DaM } & \multicolumn{2}{|c|}{$\mathrm{DaZ}$} & \multirow{2}{*}{$\begin{array}{c}\text { MANOVA } \\
\mathrm{p}\end{array}$} \\
\hline & & $M$ & $S D$ & $M$ & $S D$ & \\
\hline \multirow[t]{3}{*}{$3 ; 8$} & SUB & 93.55 & 17.04 & 58.82 & 40.45 & $.001 * *$ \\
\hline & OBJ & 71.77 & 20.14 & 32.35 & 36.19 & $.001 * *$ \\
\hline & ADJ & 66.93 & 30.54 & 20.59 & 22.07 & $.001 * *$ \\
\hline \multirow[t]{3}{*}{$4 ; 2$} & SUB & 100.00 & 0.00 & 82.35 & 30.32 & $.002 * *$ \\
\hline & OBJ & 83.87 & 21.94 & 70.59 & 29.63 & .084 \\
\hline & ADJ & 77.42 & 23.59 & 36.76 & 26.69 & $.001 * *$ \\
\hline \multirow[t]{3}{*}{$4 ; 8$} & SUB & 98.39 & 8.98 & 91.18 & 19.65 & $.087^{\mathrm{T}}$ \\
\hline & OBJ & 94.35 & 10.63 & 79.41 & 29.63 & $.014^{*}$ \\
\hline & ADJ & 79.84 & 17.58 & 66.18 & 23.29 & $.027 *$ \\
\hline \multirow[t]{3}{*}{$5 ; 2$} & SUB & 100.00 & 0.00 & 97.06 & 12.13 & .180 \\
\hline & OBJ & 91.93 & 13.52 & 75.00 & 34.23 & $.018^{*}$ \\
\hline & ADJ & 87.10 & 16.92 & 64.71 & 29.39 & $.002 * *$ \\
\hline \multirow[t]{3}{*}{$5 ; 8$} & SUB & 100.00 & 0.00 & 100.00 & 0.00 & - \\
\hline & OBJ & 94.35 & 10.62 & 89.71 & 17.81 & .262 \\
\hline & ADJ & 83.87 & 19.95 & 76.47 & 20.67 & .231 \\
\hline
\end{tabular}

** $\mathrm{p}<.01 ;{ }^{*} \mathrm{p}<.05 ;{ }^{\mathrm{T}}<.10$

Tab. 3: Mittelwerte (M) und Standardabweichung (SD) der korrekten Antworten nach Fragetyp für die DaM- und DaZ-Gruppe und Signifikanz laut MANOVA (p)

Qualität des Erwerbs. Um zu klären, ob die Erwerbsstufen und ihre Abfolge gleich sind, wurden innerhalb der DaM- und der DaZ-Gruppe jeweils die 
Interpretationsleistungen für Subjekt-, Objekt- und Adjunkt-w-Fragen miteinander verglichen. Deskriptiv ergab sich sowohl in der DaM- als auch in der DaZ-Gruppe für den Prozentsatz korrekter Antworten das erwartete Ergebnis: Subjekt $>$ Objekt $>$ Adjunkt (siehe Tabelle 3). Paarweise Vergleiche der Mittelwerte innerhalb beider Lerner-Gruppen mittels MANOVA bestätigten dieses Ergebnis im Wesentlichen. So interpretierten sowohl DaMals auch DaZ-Kinder Adjunkt-w-Fragen zu jedem Testzeitpunkt signifikant seltener korrekt als Subjekt-w-Fragen (alle $p$ s $<.05$ ).

Anhand des oben angegebenen Erwerbsalters, das auf dem MasteryKriterium von $90 \%$ korrekten Antworten basiert, lassen sich die quantitativen Unterschiede zwischen den Fragetypen auch für die Erwerbsreihenfolge bestätigen. In der DaM-Gruppe werden Subjekt-w-Fragen $(3 ; 8)$ eher als Objekt-w-Fragen (4;8) und diese eher als Adjunkt-w-Fragen beherrscht, die noch bis 5;8 nicht als sicher erworben gelten können. Die Analyse der Daten der DaZ-Gruppe ergibt die gleiche Erwerbsreihenfolge: Subjekt-wFragen $(4 ; 8)$ werden eher als Objekt-w-Fragen $(5 ; 8)$ beherrscht, und Adjunkt-w-Fragen sind bis $5 ; 8$ noch nicht sicher erworben.

Damit ergibt sich für beide Lernergruppen die gleiche Erwerbsreihenfolge: Subjekt $>$ Objekt $>$ Adjunkt.

Um zu klären, ob die Fehlermuster von DaM- und DaZ-Lernern in der jeweiligen Entwicklungsphase den gleichen Regeln unterliegen, wurde exemplarisch die Verteilung der nicht-zielsprachlichen Antworten für den ersten und den dritten Testzeitpunkt analysiert. Um eine Vergleichbarkeit der Fehlertypen zu erreichen, erfolgte jeweils eine Umrechnung der Gesamtanzahl der inkorrekten Antworten auf 100\%. In beiden Gruppen liegt jeweils nur ein geringer Anteil an nicht analysierbaren Antworten vor (zwischen $0 \%$ und 7\%). Die weiteren Fehler wurden in der Reihenfolge ihrer Annäherung an die zielsprachliche Interpretation folgenden vier Kategorien zugeordnet: keine Antwort, Ja/Nein, zusätzliche Konstituente, abweichende Konstituente (siehe Abschnitt 5.4). Bis auf den Fehlertyp ,Ja/Nein“ sind alle Kategorien in der DaM- und in der DaZ-Gruppe vertreten. Erwartungsgemäß bildet der Antworttyp ,abweichende Konstituente“, der eine spätere Erwerbsphase signalisiert, in der DaM-Gruppe die häufigste Fehlerkategorie. Ja/Nein-Antworten, die aus der Nichtinterpretation des w-Pronomens resultieren, sind in dieser Gruppe zum ersten Testzeitpunkt mit 3;8 Jahren nicht (mehr) zu beobachten. In der DaZ-Gruppe überwiegen ebenfalls die Fehler der Fehlerkategorie „abweichende Konstituente“. Der Anteil von $\mathrm{Ja} /$ Nein-Antworten beträgt zunächst $14 \%$ und geht innerhalb eines Jahres auf $2.5 \%$ zurück. Insgesamt bestätigt diese Fehleranalyse, dass sich DaM-und DaZ-Kinder in Bezug auf die Erwerbsmuster nicht wesentlich unterscheiden. Die Unterschiede in der Häufigkeit spiegeln unterschiedliche Erwerbsstadien wider. 
Einfluss externer Faktoren. In einem letzten Schritt wurde der Einfluss der externen Faktoren „,nonverbale Intelligenz“ und „Bildungshintergrund“ auf die Verstehensleistung bei w-Fragen analysiert. Die non-verbale Intelligenz wurde anhand des Gesamttestwerts des im Alter von 4;2 Jahren durchgeführten Intelligenztests K-ABC erfasst. Der Bildungshintergrund wurde, wie in der sozialwissenschaftlichen Forschung üblich, über die Schulbesuchsdauer der Mutter definiert. Zwischen der nonverbalen Intelligenz und den Leistungen im Verstehen von w-Fragen zum gleichen Testzeitpunkt ergaben sich weder in der DaM-noch in der DaZ-Gruppe statistisch signifikante Zusammenhänge (einseitige Pearson-Korrelation, DaM: $r=.192$; DaZ: $r=.401)$. Dass die Korrelation für die DaZ-Gruppe höher ausfällt als für die DaM-Gruppe, ist vermutlich darauf zurückzuführen, dass auch die nonverbalen Untertests des K-ABC nicht gänzlich sprachfrei sind. Obgleich die Mütter der DaZ-Kinder eine signifikant kürzere Schulbesuchsdauer aufweisen als die Mütter der DaM-Kinder, ergaben sich zudem wie erwartetet weder für die DaM- noch für die DaZ-Lerner Zusammenhänge zwischen dem Bildungshintergrund und der Fähigkeit, w-Fragen zu verstehen (einseitige Pearson-Korrelationen für 4;2 Jahre, DaM: $r=.072$; DaZ: $r=-.049)$.

\section{Diskussion: Wer versteht wann was?}

Die Fragen nach der Erwerbsgeschwindigkeit (8a) lassen sich auf der Grundlage der in Abschnitt 5.6 dargestellten Ergebnisse wie folgt beantworten: Im Alter von 5;8 haben frühe Zweitsprachlerner im Wesentlichen die zielsprachliche Interpretation von w-Fragen gemeistert. Damit liegen sie im Vergleich mit monolingualen Lernern lediglich ein Jahr zurück. Das gleiche Bild ergibt sich für die Beherrschung der verschiedenen Subtypen: Subjektw-Fragen werden von den DaZ-Kindern mit 4;8 (DaM: 3;8) und Objekt-wFragen mit 5;8 (DaM: 4;8) beherrscht; Adjunkt-w-Fragen werden weder in der DaZ- noch in der DaM-Gruppe bis zum Alter von 5;8 sicher zielsprachlich interpretiert. Im Vergleich zu den monolingualen Altersgenossen werden die einzelnen Erwerbsstufen in der Interpretation von w-Fragen - legt man die Kontaktdauer zum Deutschen zugrunde - rascher durchlaufen. Dieser Erwerbsverlauf bestätigt die querschnittlichen Ergebnisse bisheriger Studien zur zielsprachlichen Interpretation von w-Fragen im monolingualen Erwerb (Penner 1999; Siegmüller/Herzog/Herrmann 2005) und im frühen Zweitsprach-Erwerb (Schulz/Tracy/Wenzel 2008). Darüber hinaus findet sich, anders als von Penner (2003) konstatiert, im Bereich des w-FrageVerstehens keinerlei Evidenz für eine Stagnation im frühen Zweitspracherwerb. Die hohe Erwerbsgeschwindigkeit entspricht den Befunden für den Erwerb der Satzstruktur in der Sprachproduktion (Tracy/Thoma 2009). 
Die Untersuchungsfragen nach der Qualität des Erwerbs (8b) lassen sich wie folgt beantworten: In der DaM- und der DaZ-Gruppe sind die Erwerbsstufen identisch. Subjekt-w-Fragen werden jeweils vor Objekt-w-Fragen und diese vor Adjunkt-w-Fragen beherrscht; auch der jeweilige Anteil korrekter Antworten entspricht dieser Erwerbsreihenfolge. Eine Analyse der Fehlermuster in beiden Lernergruppen bestätigt, dass die gleichen Fehlertypen $\mathrm{zu}$ beobachten sind und dass diese in der jeweiligen Entwicklungsphase den gleichen Regeln unterliegen. Zu Beginn des w-Fragen-Erwerbs sind Ja/ Nein-Antworten zu beobachten, die aus der Nicht-Interpretation des wFragepronomens resultieren; diese sind nur in der DaZ-Gruppe attestiert. Die Antwort mit einer inkorrekten Konstituente entspricht dem fortschrittlichsten Fehlermuster; dieses ist in der DaM-Gruppe häufiger als in der DaZ-Gruppe festzustellen.

Die Ergebnisse dieser Studie zum w-Frage-Verstehen liefern weitere Evidenz für Parallelen zwischen frühem Zweitspracherwerb und monolingualem Spracherwerb. Dass die Interpretation von w-Fragen Teil der spezifisch sprachlichen, kerngrammatischen Kompetenz ist, lässt sich schließlich daraus ableiten, dass keine Korrelation zwischen der Fähigkeit zur Interpretation von w-Fragen und externen Faktoren wie Bildungshintergrund und nonverbale Intelligenz gefunden wurde.

\section{Fazit}

Frühe Zweitsprachlerner interpretieren einfache w-Fragen mit ca. fünfeinhalb Jahren zielsprachlich, nach knapp drei Jahren Kontakt mit dem Deutschen und chronologisch nur ca. ein Jahr später als monolinguale Sprachlerner. Damit bestätigt sich das für den Morpho-Syntax-Erwerb in der Sprachproduktion gefundene Erwerbsmuster: Bei einem frühen Erwerbsbeginn ist keine Stagnation des Spracherwerbs, sondern im Gegenteil ein rascheres Durchlaufen der Erwerbsphasen zu beobachten. Auch in Bezug auf die Erwerbsreihenfolge und die nicht-zielsprachlichen Antwortmuster für w-Fragen verhalten sich frühe Zweitsprachlerner wie einsprachige Lerner.

Zwei Schlüsse lassen sich für die Spracherwerbsforschung aus diesen Ergebnissen ziehen: Das Ende der kritischen Phase für den erfolgreichen Erwerb der w-Fragen liegt nicht vor dem dritten Geburtstag. Zudem scheinen die typologischen Unterschiede zwischen w-Fragen im Deutschen und den verschiedenen Erstsprachen der DaZ-Kinder nicht zu einem negativen Transfer für die korrekte Interpretation von w-Fragen zu führen.

Für Sozialwissenschaften und Bildungspolitik folgt aus diesen Ergebnissen: Geht es um Sprache, sollte statt Migrationshintergrund der Spracherwerbstyp der Kinder erfasst werden; das setzt detaillierte Informationen 
zur Spracherwerbsbiographie der Lerner voraus. Um vorhandene Sprachfähigkeiten der frühen Zweitsprachlerner und Erwerbsfortschritte adäquat abbilden zu können, müssen bei altersparallelisierten Erhebungen zusätzlich das Alter bei Erwerbsbeginn und die Kontaktdauer berücksichtigt werden.

Das hier gezeichnete optimistische Bild für die Erfolgsaussichten des frühen Zweitspracherwerbs sollte jedoch über Dreierlei nicht hinwegtäuschen: dass für andere Erwerbsphänomene frühere kritische Perioden gelten können, dass für den späteren Bildungserfolg die Beherrschung der kerngrammatischen Bereiche Pflicht ist und nicht die Kür, die automatisch den gelungenen Aufsatz erzeugt, und dass die im deutschen Bildungssystem immer noch starke Abhängigkeit des Schulerfolgs von der sozialen Herkunft (vgl. Klieme et al. 2010) häufig eine Bildungsbarriere darstellt, die unabhängig von der Sprache wirkt - für Kinder mit Migrationshintergrund, für frühe Zweitsprachlerner und für die späteren Migrantendeutschen.

\section{Literatur}

Ahrenholz, Bernt (Hg.) (2006): Kinder mit Migrationshintergrund - Spracherwerb und Fördermöglichkeiten. Freiburg i.Br.

Bos, Wilfried et al. (2007): IGLU 2006. Lesekompetenzen von Grundschulkindern in Deutschland im internationalen Vergleich. Münster u.a.

Bialystok, Ellen (1997): The structure of age: in search of barriers to second language acquisition: In: Second Language Research 13, 2, S. 116-137.

Chilla, Solveig (2008): Erstsprache, Zweitsprache, Spezifische Sprachentwicklungsstörung? Eine Untersuchung des Erwerbs der deutschen Hauptsatzstruktur durch sukzessiv-bilinguale Kinder mit türkischer Erstsprache. (= Schriftenreihe Philologia 124). Hamburg.

Chilla, Solveig/Bonnesen, Matthias (2011): A crosslinguistic perspective on child SLA. The acquisition of questions in German and French. In: Linguistische Berichte 228, S. 413-442.

Chomsky, Noam (1986): Knowledge of language: its nature, origin, and use. London.

Dayal, Veneeta (2002): Single-pair vs. Multiple-pair answers: wh in-situ and scope. In: Linguistic Inquiry 33, 3, S. 512-520.

De Houwer, Annick (1995): Bilingual language acquisition. In: Fletcher, Paul/MacWhinney, Brian (Hg.): The handbook of child language. (= Blackwell Handbooks in Linguistics 2). Oxford, S. 219-250.

Demuth, Katherine (1996): Collecting spontaneous production data. In: McDaniel/ McKee/Cairns (Hg.), S. 3-22.

De Villiers, Jill G./Roeper, Tom (1996): Questions after stories: on supplying context and eliminating it as a variable. In: McDaniel/McKee/Cairns (Hg.), S. 163-187. 
Friedmann, Naama/Novogrodsky, Rama (2011): Which questions are most difficult to understand? The comprehension of wh-questions in three subtypes of SLI. In: Lingua 121, S. 367-382.

Grewendorf, Günther (2002): Minimalistische Syntax. Tübingen/Basel.

Grimm, Angela et al. (einger.): Die Rolle interner und externer Faktoren auf grammatische Fähigkeiten im Erst- und frühen Zweitspracherwerb.

Grimm, Angela/Schulz, Petra (2012): Forschungsmethoden der kombinierten Längsund Querschnittsstudie MILA. In: Ahrenholz, Bernt (Hg.): Einblicke in die Zweitspracherwerbsforschung und ihre methodischen Verfahren. (= DaZ-Forschung. Deutsch als Zweitsprache, Mehrsprachigkeit und Migration 1). Berlin/Boston, S. 195-218.

Grimm, Hannelore (Hg.) (2000): Sprachentwicklung. Enzyklopädie der Psychologie (Themenbereich C, Theorie und Forschung: Ser. 3, Sprache). Bd. 3. Göttingen.

Guasti, Maria Theresa (2002): Language acquisition. The growth of grammar. Cambridge, MA.

Haberzettl, Stefanie (2005): Der Erwerb der Verbstellungsregeln in der Zweitsprache Deutsch durch Kinder mit russischer und türkischer Muttersprache. (= Linguistische Arbeiten 495). Tübingen.

Hopp, Holger (2011): Internal and external factors in the child L2 development of the German determiner phrase. In: Linguistic Approaches to Bilingualism 1, 3, S. 238264.

Hyltenstam, Kenneth (1992): Non-native features of near-native speakers. On ultimate attainment of childhood L2 learners. In: Harris, Richard, J. (Hg.): Cognitive processing in bilinguals. (= Advances in Psychology 83). Amsterdam, S. 351-368.

Klieme, Eckhard et al. (2010): PISA 2009: Bilanz nach einem Jahrzehnt. Münster.

Knapp, Werner (1999): Verdeckte Sprachschwierigkeiten. In: Die Grundschule 31, 5, S. 30-33.

Krashen, Stephen D. (1973): Lateralization, language learning and critical period. Some new evidence. In: Language Learning 23, S. 63-74.

Lemke, Vytautas (2009): Der Erwerb der DP: Variation im frühen Zweitspracherwerb. Dissertation. Universität Mannheim.

Locke, John L. (1997): A theory of neurolinguistic development. In: Brain and Language 58, S. 265-326.

Long, Martyn (1990): Maturational constraints on language development. In: Studies in Second Language Acquisition 12, S. 251-285.

Marinis, Theodoros/Chondrogianni, Vicky (2011): Comprehension of reflexives and pronouns in sequential bilingual children: do they pattern similarly to L1 children, L2 adults, or children with specific language impairment? In: Journal of Neurolinguistics 24, 2, S. 202-212.

McDaniel, Dana/McKee, Cecile/Cairns, Helen (Hg.) (1996): Methods for assessing children's syntax. (= Language, Speech, and Communication). Cambridge, MA. 
Meisel, Jürgen (2009): Second language acquisition in early childhood. In: Zeitschrift für Sprachwissenschaft 28, S. 5-34.

Melchers, Peter/Preuß, Ulrich (2003): Kaufman-Assessment Battery for Children: $\mathrm{K}-\mathrm{ABC}$. Individualtest zur Messung von Intelligenz und Fertigkeiten bei Kindern im Alter von 2;6 bis 12;5 Jahren. Durchführungs- und Auswertungshandbuch. 6. Aufl., deutschsprachige Fassung. Leiden. [Original von Alan S. Kaufman und Nadeen L. Kaufman.]

Nicholas, Howard/Lightbown, Patsy M. (2008): Defining child second language acquisition, defining roles for instruction. In: Philp, Jenefer/Oliver, Rhonda/ Mackey, Alison (Hg.). Second language acquisition and the younger learner: child's play? (= Language Learning and Language Teaching 23 ). Amsterdam, S. 27-51.

Ose, Julia/Schulz, Petra (2010): Was fehlt Jonas - Ein Taschentuch oder das Taschentuch? Eine Pilotstudie zum Artikelerwerb bei Kindern mit Deutsch als Zweitsprache. In: Rost-Roth, Martina (Hg.): DaZ - Spracherwerb und Sprachförderung Deutsch als Zweitsprache. Beiträge aus dem 5. Workshop Kinder mit Migrationshintergrund. Freiburg i.Br., S. 79-97.

Penner, Zvi (1994): Asking questions without CPs? On the acquisition of wh questions in Bernese Swiss German and Standard German. In: Hoekstra, Teun/Schwartz, Bonnie (Hg.): Language acquisition studies in Generative Grammar. (= Language Acquisition \& Language Disorders 8). Amsterdam, S. 177-214.

Penner, Zvi (1998): Sprachentwicklung und Sprachverstehen bei Ausländerkindern. Eine Pilotstudie bei Schulkindern in der deutschen Schweiz. In: Wegener, Heide (Hg.): Eine zweite Sprache lernen. Empirische Untersuchungen zum Zweitspracherwerb. (= Tübinger Beiträge zur Linguistik: Serie A; 24). Tübingen, S. 241261.

Penner, Zvi (1999): Screening-Verfahren zur Feststellung von Störungen in der Grammatikentwicklung. Gebrauchsanweisung für die BenutzerInnen. (= Schriftenreihe des Heilpädagogischen Seminars Zürich 14A). Luzern.

Penner, Zvi (2003): Neue Wege der sprachlichen Förderung von Migrantenkindern. (= Forschung für die Praxis). Berg, Thurgau.

Rauch, Wolfgang/Grimm, Angela/Schulz, Petra (einger.): Der Zusammenhang von Arbeitsgedächtnis und grammatischen Fähigkeiten im Erst- und frühen Zweitspracherwerb.

Rizzi, Luigi (1996): Residual verb second and the wh-criterion. In: Belletti, Adriana/ Rizzi, Luigi (Hg.): Parameters and functional heads. Essays in comparative syntax. (= Oxford Studies in Comparative Syntax). New York, S. 63-90.

Rothweiler, Monika (2007): Multilingualism and specific language impairment. In: Auer, Peter/Wei, Li (Hg.): Handbook of multilingualism and multilingual communication. (= Handbooks of Applied Linguistics 5). Berlin/New York, S. 229-246.

Rothweiler, Monika (2009): Critical periods and SLI. Comment on Jürgen Meisel „Second language acquisition in early childhood“. In: Zeitschrift für Sprachwissenschaft 28, S. 49-57. 
Schönenberger, Manuela/Rothweiler, Monika/Sterner, Franziska (2012): Case marking in child L1 and early child L2 German. In: Gabriel, Christoph/Braunmüller, Kurt (Hg.) Multilingual individuals and multilingual societies (= Hamburg Studies on Multilingualism 13). Amsterdam, S. 3-22.

Schulz, Petra (2007): Erstspracherwerb Deutsch: Sprachliche Fähigkeiten von Eins bis Zehn. In: Graf, Ulrich/Moser Opitz, Elisabeth (Hg.): Diagnostik und Förderung im Elementarbereich und Grundschulunterricht. Lernprozesse wahrnehmen, deuten und begleiten. (= Entwicklungslinien der Grundschulpädagogik 4). Baltmannsweiler, S. 67-86.

Schulz, Petra (2012): Why and how individual differences matter for linguistic theory and experimental research but not for UG. In: Linguistic Approaches to Bilingualism 2, 3, S. 298-303.

Schulz, Petra/Grimm, Angela (2012) Spracherwerb. In: Drügh, Heinz et al. (Hg.): Germanistik. Sprachwissenschaft - Literaturwissenschaft - Schlüsselkompetenzen. Stuttgart/Weimar, S. 155-172.

Schulz, Petra/Roeper, Tom (2011): Acquisition of exhaustivity in wh-questions: a semantic dimension of SLI? In: Lingua 121, 3, S. 383-407.

Schulz, Petra/Tracy, Rosemarie (2011): Linguistische Sprachstandserhebung - Deutsch als Zweitsprache (LiSe-DaZ). (= Hogrefe-Vorschultests). Göttingen.

Schulz, Petra/Tracy, Rosemarie/Wenzel, Ramona (2008): Linguistische Sprachstandserhebung - Deutsch als Zweitsprache (LiSe-DaZ): Theoretische Grundlagen und erste Ergebnisse. In: Ahrenholz, Bernt (Hg.): Zweitspracherwerb: Diagnosen, Verläufe, Voraussetzungen. Freiburg i.Br., S. 17-42.

Schwartz, Bonnie D. (2003): Child L2 acquisition: paving the way. In: Beachley, Barbara/Brown, Amanda/Colin, Frances (Hg.): BUCLD 27: Proceedings of the 27th Annual Boston University Conference of Language Development. Sommerville, MA, S. 26-50.

Siegmüller, Julia/Herzog, Carmen/Herrmann, Heike (2005): Syntaktische und lexikalische Aspekte beim Verstehen von Informationsfragen - ein Vergleich zwischen Kindern mit spezifischer Sprachentwicklungsstörung und Kindern mit ungestörter Sprachentwicklung. In: L.O.G.O.S. interdisziplinär 13, S. 29-35.

Thoma, Dieter/Tracy, Rosemarie (2006): Deutsch als frühe Zweitsprache: zweite Erstsprache? In: Ahrenholz (Hg.), S. 58-79.

Tracy, Rosemarie (1994): Raising questions: formal and functional aspects of the acquisition of wh-questions in German. In: Tracy, Rosemarie/Lattey, Elsa (Hg.): How tolerant is Universal Grammar: essays on language learnability and language variation. (= Linguistische Arbeiten 309). Tübingen, S. 1-34.

Tracy, Rosemarie (2000): Sprache und Sprachentwicklung: Was wird erworben? In: Grimm (Hg.), S. 3-39.

Tracy, Rosemarie (2008): Wie Kinder Sprachen lernen. Und wie wir sie dabei unterstützen können. 2., überarb. Aufl. Tübingen.

Tracy, Rosemarie/Gawlitzek-Maiwald, Ira (2000): Bilingualismus in der frühen Kindheit. In: Grimm (Hg.), S. 495-535. 
Tracy, Rosemarie/Thoma, Dieter (2009): Convergence on finite clauses in L1, bilingual L1 and early L2 acquisition. In: Dimroth, Christine/Jordens, Peter (Hg.): Functional categories in learner language. (= Studies on Language Acquisition 37). Berlin/New York, S. 1-43.

Tracy, Rosemarie/Lemke, Vytautas (2012): Young L2 and L1 learners: more alike than different. In: Watorek, Marzena/Benazzo, Sandra/Hickmann, Maya (Hg.): Comparative perspectives on language acquisition - a tribute to Clive Perdue. (= Second Language Acquisition 61). Bristol, S. 303-323.

Unsworth, Sharon (2005): Child L2, adult L2, child L1: differences and similarities. A study on the acquisition of object scrambling in Dutch. Utrecht.

Wenzel, Ramona/Schulz, Petra/Tracy, Rosemarie (2009): Herausforderungen und Potential der Sprachstandsdiagnostik - Überlegungen am Beispiel von LiSe-DaZ. In: Reich, Hans H./Roth, Hans-Joachim (Hg.): Dokumentation der FörMig-Herbsttagung 2007: Von der Sprachdiagnose zur Sprachförderung. (= FörMig-Edition 5). Münster u.a., S. 45-70.

White, Lydia (2000): Second language acquisition: from initial to final state. In: Archibald, John (Hg.): Second language acquisition and linguistic theory. Malden, MA, S. 130-150. 
